\title{
Organic electro-optic glasses for WDM applications
}

Larry Dalton, Axel Scherer, Antao Chen, Alex Jen, Philip Reid, et al.

Larry Dalton, Axel Scherer, Antao Chen, Alex Jen, Philip Reid, Bruce Robinson, Bruce Eichinger, M. Hochberg, T. Baehr-Jones, Anna Pyajt, Jocelyn Takayesu, Philip Sullivan, Andrew Akelaitis, Rhys Lawson, Denise Bale, Marnie Haller, Jingdong Luo, Sen Liu, Yi Liao, Kimberly Firestone, Sanchali Bhattacharjee, Jessica Sinness, Scott Hammond, Allyson Sgro, Nicholas Buker, Robert Snoeberger, Mark Lingwood, William Steier, "Organic electro-optic glasses for WDM applications," Proc. SPIE 6014, Active and Passive Optical Components for WDM Communications V, 60140P (24 October 2005); doi: 10.1117/12.634366 


\title{
Organic Electro-Optic Glasses for WDM Applications
}

\author{
Larry Dalton*a,b ${ }^{*}$ Axel Scherer ${ }^{\mathrm{c}}$, Antao Chen ${ }^{\mathrm{d}}$, Alex Jen ${ }^{\mathrm{a}, \mathrm{e}}$, Philip Reid ${ }^{\mathrm{a}}$, Bruce Robinson ${ }^{\mathrm{a}}$, Bruce Eichinger ${ }^{\mathrm{a}}, \mathrm{M}$. \\ Hochberg $^{\mathrm{c}}$, T. Baehr-Jones ${ }^{\mathrm{c}}$, Anna Pyajt ${ }^{\mathrm{d}}$, Jocelyn Takayesu ${ }^{\mathrm{a}}$, Philip Sullivan ${ }^{\mathrm{a}}$, Andrew Akelaitis ${ }^{\mathrm{a}}$, Rhys Lawson ${ }^{\mathrm{a}}$, Denise \\ Bale $^{\mathrm{a}}$, Marnie Haller ${ }^{\mathrm{b}}$, Jingdong Luo ${ }^{\mathrm{e}}$, Sen Liu ${ }^{\mathrm{e}}$, Yi Liao ${ }^{\mathrm{a}}$, Kimberly Firestone ${ }^{\mathrm{a}}$, Sanchali Bhattacharjee ${ }^{\mathrm{a}}$, Jessica Sinness ${ }^{\mathrm{a}}$, \\ Scott Hammond ${ }^{\mathrm{a}}$, Allyson Sgro ${ }^{\mathrm{a}}$, Nicholas Buker ${ }^{\mathrm{a}}$, Robert Snoeberger ${ }^{\mathrm{a}}$, Mark Lingwood ${ }^{\mathrm{a}}$, and William Steier ${ }^{\mathrm{f}}$ \\ ${ }^{\mathrm{a}}$ Department of Chemistry, ${ }^{\mathrm{b}}$ Department of Electrical Engineering, ${ }^{\mathrm{d}}$ Applied Physics Laboratory, and ${ }^{\mathrm{e}}$ Department of \\ Materials Science \& Engineering, and University of Washington, Seattle, WA 98195 \\ ${ }^{\mathrm{c}}$ Department of Applied Physics, California Institute of Technology, Pasadena, CA 91125 \\ ${ }^{\mathrm{f}}$ Department of Electrical Engineering, University of Southern California, Los Angeles, CA 90089
}

\begin{abstract}
This communication primarily deals with utilizing organic electro-optic (OEO) materials for the fabrication of active wavelength division multiplexing (WDM) transmitter/receiver systems and reconfigurable optical add/drop multiplexers (ROADMs), including the fabrication of hybrid OEO/silicon photonic devices. Fabrication is carried out by a variety of techniques including soft and nanoimprint lithography. The production of conformal and flexible ring microresonator devices is also discussed. The fabrication of passive devices is also briefly reviewed. Critical to the realization of improved performance for devices fabricated from OEO materials has been the improvement of electro-optic activity to values of $300 \mathrm{pm} / \mathrm{V}$ (or greater) at telecommunication wavelengths. This improvement in materials has been realized exploiting a theoretically-inspired (quantum and statistical mechanics) paradigm for the design of chromophores with dramatically improved molecular first hyperpolarizability and that exhibit intermolecular electrostatic interactions that promote self-assembly, under the influence of an electric poling field, into noncentrosymmetric macroscopic lattices. New design paradigms have also been developed for improving the glass transition of these materials, which is critical for thermal and photochemical stability and for optimizing processing protocols such as nanoimprint lithography. Ring microresonator devices discussed in this communication were initially fabricated using chromophore guest/polymer host materials characterized by electro-optic coefficients on the order of $50 \mathrm{pm} / \mathrm{V}$ (at telecommunication wavelengths). Voltage-controlled optical tuning of the pass band of these ring microresonators was experimental determined to lie in the range 1-10 GHz/V or all-organic and for $\mathrm{OEO} /$ silicon photonic devices. With new materials, values approaching 50 $\mathrm{GHz} / \mathrm{V}$ should be possible. Values as high as $300 \mathrm{GHz} / \mathrm{V}$ may ultimately be achievable.
\end{abstract}

Keywords: Electro-optic ring microresonators, silicon photonics, molecular first hyperpolarizability, wavelength division multiplexing, reconfigurable optical add/drop multiplexing, optical rectification, organic EO materials

\section{Introduction}

Organic electro-optic (OEO) materials and devices have been of interest for more than a decade because of the potential for high bandwidth, low drive voltage operation, and because of their adaptability to a range of processing protocols for fabrication of a wide array of novel devices including phased array antennae, optical gyroscopes, and optochips. ${ }^{1-9}$ Devices fabricated from organic materials afford an important advantage over devices based on inorganic materials (such as lithium niobate) for high speed/broad bandwidth operation. ${ }^{1-13}$ Low dielectric constants, low dispersion, and fast electronic response, associated with the $\pi$-electrons of organic materials, permit high frequency operation, including over the full $\mathrm{W}$ band $(75-110 \mathrm{GHz})$ range. ${ }^{10,11}$ Organic electro-optic materials have also proven attractive for terahertz signal generation and detection and may be of utility for terahertz imaging. ${ }^{12,13}$

A characteristic of organic electro-optic materials that has become more recently appreciated is their adaptability to a variety of processing methodologies. Thin film devices can be fabricated both by solution (e.g., spin casting and sequential synthesis ${ }^{14,15}$ ) and vapor deposition (including sequential synthesis protocols). Conformal and flexible devices have been fabricated by lift-off techniques with properties remaining essentially unchanged even under extreme and repeated flexing and bending. ${ }^{16}$ Stripline (Mach Zehnder) and ring microresonator devices have been fabricated by soft and nanoimprint lithography suggesting the potential for low cost, mass production of complex circuitry. ${ }^{17-19}$ Organic electro-optic materials have been incorporated into silicon photonic devices including ring micro-

*dalton@chem.washington.edu; phone 1206 543-1686; fax. 1206 685-8665

Active and Passive Optical Components for WDM Communications V, edited by Achyut K. Dutta, Yasutake Ohishi, Niloy K. Dutta, Jesper Moerk, Proc. of SPIE Vol. 6014, 60140P, (2005) · 0277-786X/05/\$15 · doi: 10.1117/12.634366 
resonators, slotted ring microresonators, and photonic bandgap structures. ${ }^{20,21}$ Frequency tuning of $7 \mathrm{GHz} / \mathrm{V}$ has been demonstrated with silicon slotted ring microresonators filled with organic electro-optic materials. ${ }^{20}$ High bandwidth optical rectification was also observed with these hybrid slotted ring microresonators. ${ }^{20}$ Vertical integration with very large scale integration (VLSI) semiconductor electronics and integration with silica fiber optics has been achieved. ${ }^{6,22-30}$ Three-dimensional (3D) electro-optic circuitry has been fabricated and indeed fabricated on top of VLSI wafers. ${ }^{6-9,22-30}$ 3D (vertical slope) fabrication techniques have also been employed to produce electro-optic wedges (analogous to in-line fiber modulator structures) on top of low optical loss transmission fibers. ${ }^{6-9,22-30}$ Such structures permit low (e.g., less than $1 \mathrm{~dB}$ per connection) optical loss coupling of light into and out of electro-optic devices structures. Utilization of tapered transitions, wedge structures, or spherical lens has permitted total insertion loss to be reduced to values of $6 \mathrm{~dB}$ or less. Such values are approaching those obtained for lithium niobate devices but lithium niobate can always be expected to afford an advantage of lower material loss. With lithium niobate, material loss is insignificant $(\sim 0.2 \mathrm{~dB} / \mathrm{cm})$ and insertion loss is limited by index matching rather than mode size matching. A variety of novel device structures, including stripline, ${ }^{31-34}$ cascaded prism, ${ }^{35}$ all-organic ring microresonator, ${ }^{17-19,36-46}$ and $\mathrm{OEO} /$ silicon photonic ring microresonator ${ }^{20,21}$ structures have been fabricated from organic electro-optic materials.

The potential for organic electro-optic materials to exhibit large electro-optic coefficients (tensor elements $r_{33}$ and $\mathrm{r}_{13}$ for poled polymer materials) has been appreciated for more than a decade but it is only within the last five years that values have exceeding those of lithium niobate $(\sim 30 \mathrm{pm} / \mathrm{V})$ at telecommunication wavelengths have been achieved. ${ }^{31}$ In the past year, electro-optic activities of greater than $300 \mathrm{pm} / \mathrm{V}$ have been realized for organic materials at telecommunication wavelengths. ${ }^{47-50}$ One objective of this communication is to provide insight into how these high values were achieved and to speculate on what further improvements can be realized in the near term. It is also important to discuss auxiliary properties such as optical loss, thermal stability, photochemical stability, and processability. However, before we turn our attention to a discussion of improvement of electro-optic activity by theoretically-inspired rational design, it is useful to discuss the motivation for achieving large electro-optic coefficients. Frequently, the motivation is expressed in terms of the relationship of electro-optic activity to device drive voltages (e.g., $\mathrm{V}_{\pi}$ in the case of Mach Zehnder modulators). ${ }^{31}$ In the field of rf photonics, the motivation is to achieve transparent (lossless) telecommunication links. Even better would be to achieve gain in the electrical-optical-electrical signal transduction process. The drive voltage required to achieve transparency in telecommunication links employing stripline EO devices is nominally on the order of 0.5 volts. For a Mach Zehnder modulator: $\mathrm{V}_{\pi}=\lambda \mathrm{h} /\left(\mathrm{Cn}^{3} \mathrm{r}_{33} \mathrm{~L} \Gamma\right)$ where $\lambda$ is the operational wavelength, $h$ is the drive electrode spacing, $n$ is the index of refraction, $L$ is the optical/electrical field interaction (device) length, $\Gamma$ is the modal overlap parameter, and $\mathrm{C}=1$ or 2 depending on whether single arm or pushpull operation is utilized. It is clear from this expression that the relationship between $V_{\pi}$ and $r_{33}$ will depend on device design. Moreover, other critical performance parameters, such as bandwidth and insertion loss, will be defined by device design. For example, resistive losses in metal electrodes typically limit bandwidth in stripline devices. ${ }^{10,11}$ If one assumes the resistive loss of gold, a waveguide loss of the electro-optic materials of $2 \mathrm{~dB} / \mathrm{cm}$, an electro-optic coefficient of $300 \mathrm{pm} / \mathrm{V}$, a $0.5 \mathrm{~cm}$ electrode structure with a low loss mode transformer results in a $3 \mathrm{~dB}$ operational bandwidth of approximately $100 \mathrm{GHz}$, an insertion loss of $3 \mathrm{~dB}$, and a $\mathrm{V}_{\pi}$ of 0.75 volts. Clearly, there is a motivation to achieve electro-optic coefficients even greater than $300 \mathrm{pm} / \mathrm{V}$. Cox and coworkers ${ }^{34}$ emphasize that the traditional focus has been oversimplified and that the strong motivation for higher electro-optic activity also relates to optical power, bandwidth, and device size considerations. We also call to the reader's attention to the fact that it may be desirable to trade bandwidth for size and low drive voltages by using device structures such as ring microresonators rather than stripline devices for electrical-to-optical signal transduction and data management (e.g., optical switching). An example of such trade-off is the exceptional performance of hybrid organic EO/silicon photonic split ring microresonators. Indeed, the second major topic of this communication (the first being optimization of material properties) is investigation of the application of ring microresonators for active WDM and ROADM applications.

In this communication, we restricted our consideration of organic electro-optic thin film materials prepared by electric field poling of dipolar charge transfer chromophores. However, our consideration of the role played by intermolecular electrostatic interactions is relevant to understanding the formation of acentric organic crystals and for the order observed in thin films prepared by sequential (Langmuir-Blodgett or Merrifield-type) synthesis from functionalized surfaces.

Our communication is organized into two parts: (1) Optimizing material properties and (2) exploration of allorganic and $\mathrm{OEO} /$ silicon photonics hybrid ring microresonators for applications related to telecommunications. The first part will hopefully provide the reader with an idea of improvements in performance that can be expected in the near future. 


\section{Optimizing Material Electro-Optic Activity and Auxiliary Properties}

Macroscopic electro-optic activity is related to molecular electro-optic activity (molecular first hyperpolarizability $\beta$ ) by the following expression: $r_{33}=2 \mathrm{~N} \beta f(\omega)<\cos ^{3} \theta>/ n^{4}$ where $n$ is the index of refraction, $N$ is the chromophore number density in the material, $f(\omega)$ is a local field factor accounting for the dielectric nature of the material around the chromophore, and $\left\langle\cos ^{3} \theta\right\rangle$ is the electric field poling-induced chromophore acentric order parameter. $\mathrm{N}$ and $\left\langle\cos ^{3} \theta>\right.$ are not independent parameters because of intermolecular electrostatic interactions. Thus, optimizing macroscopic electro-optic activity can be divided into two operations: (1) Use of quantum mechanics to optimize $\beta$ and (2) use of statistical mechanics to optimize the product $\mathrm{N}<\cos ^{3} \theta>$.

The use of single molecule quantum mechanics relies on the absence of orbital interactions between chromophores. Such isolation is desired for other reasons including to avoid charge transport (conductivity) between chromophores that would lead to reduction in the poling field felt by chromophores and to bias voltage drift in operational devices.

There are two classes of chromophore modifications that are normally considered when attempting to try to improve chromophore $\beta$ values. The first is the obvious realization of maximum molecular first hyperpolarizability by the systematic optimization of individual donor, bridge, and acceptor moieties of charge-transfer type chromophores. The second is to explore novel chromophore architectures such as " $\mathrm{X}$ " shaped ${ }^{51-53}$ chromophores and twisted $^{48,54-55}$ chromophores. The first question that must be asked is "How reliable are various quantum mechanical methods in predicting desired structure/function trends?" If one is speaking of simple modification of donor, bridge, and acceptor moieties, the answer (based on comparing the results of Hartree-Fock, Density Functional Theory (B3LYP and PBE), and INDO methods for calculating trends in the variation of molecular first hyperpolarizability for a series of 12 chromophores) seems to be that quantum mechanical methods do a reasonable job of predicting changes in $\beta$ with simple donor, bridge, and acceptor modifications. ${ }^{48}$ A recent article by Robinson and coworkers ${ }^{56}$ examines the reliability of density functional theory (DFT) calculations for predicting changes in $\beta$ with variation of bridge and acceptor groups for a series of chromophores based on ferrocenyl donors. Excellent agreement is obtained with experimentally-defined (by wavelength-agile, femtosecond Hyper-Rayleigh Scattering (HRS) measurements) relative (to $\mathrm{p}$-nitroaniline) $\beta$ values and theoretically-calculated $\beta$ values (referenced to $\mathrm{p}$-nitroaniline). Referencing results to $\mathrm{p}$ nitroaniline was effected to avoid dielectric medium effects complicating comparison of theory and experiment. Moreover, it is important to keep in mind that one is ultimately interested in trends when designing new chromophores rather than in absolute $\beta$ values. In reference 48, other comparisons between theory and experiment are given (considering the variation of $\beta$ with changes in acceptor structure for amine donor/vinyl bridge structures) reinforcing the conclusions of reference 56. Theory predicts that $\beta$ will increase with replacement of the methyl group of the tricyanovinylfuran (TCF) acceptor with a trifluromethyl group. This trend has been repeatedly observed experimentally and has been exploited by researchers at Lockheed Martin and Corning as well as by our group. Replacement of the furan ring with a pyrroline ring is predicted to lead to even greater improvement. This observation is qualitatively confirmed by HRS measurements. In like manner, pyrrolizine acceptors have been found to lead to improved molecular hyperpolarizability. It should be noted that in this work we discussed $\beta_{\mathrm{HRS}}$ values because we are focused on comparing theoretical results with the results of HRS experiments. It is the component of $\beta$ along the dipole moment vector that is relevant to macroscopic electro-optic activity induced by electric field poling. It is also common to report theoretical

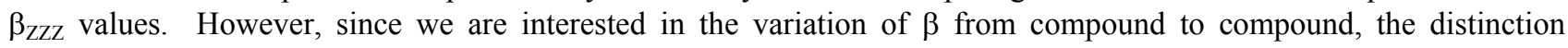
between these different elements of $\beta$ is relatively unimportant. It is clear that for simple chromophore modification discussed to this point, various quantum mechanical methods are effectively in predicting trends (relative $\beta$ values). This is not always the case as is illustrated for the case of twisted chromophores. ${ }^{48}$ Various methods fail to agree on the magnitude for even the sign of maximum molecular hyperpolarizability as a function of twist angle. ${ }^{48}$ While the different predictions of various methods can be rationalized (singularities and the role of parameterization of semiempirical methods), it is clear that the reliability of theoretical methods is not good for twisted chromophores. Reasonable agreement is observed between various theoretical methods for X-shaped chromophores.

In summary, quantum mechanical calculations, while not always reliable predictors of changes in $\beta$ with changing chromophore structure, have in most cases provided very valuable guidance. Improvement of $\beta$ has played an important role in the realization of state-of-the-art electro-optic activities and further significant (perhaps as much as an order of magnitude) improvement in $\beta$ can be anticipated. A point that must be kept in mind in improving $\beta$ is the optical nonlinearity/optical transparency trade-off. For neutral ground state chromophores, improving $\beta$ normally results in some red shifting of the absorption maximum of the interband charge transfer transition. Care must be exercised that interband absorption loss at 1.3 microns telecommunications wavelength does not increase to values greater than the 
absorption loss contributed by hydrogen overtone vibrational transitions. ${ }^{57,58}$ Interband electronic absorption is typically not a problem for operation at 1.55 mcirons. $\mathrm{X}$-shaped chromophores are interesting in that an increase in $\beta$ is accompanied by a blue-shifting of the interband absorption maximum. However, in preliminary studies these chromophores have been found to aggregate and thus must be modified to inhibit such aggregation before chromophores can be exploited for realization of improved electro-optic activity.

If intermolecular electrostatic static interactions are treated in the point dipole approximation ${ }^{59}$ (neglecting nuclear repulsive interactions or chromophore shape effects), the acentric order parameter is calculated to be $\left\langle\cos ^{3} \theta>=\right.$ $\mu \mathrm{F} / 5 \mathrm{kT}\left[1-\mathrm{L}^{2}(\mathrm{~W} / \mathrm{kT})\right]$ where $\mu$ is the chromophore dipole moment, $\mathrm{F}$ is the effective poling field (corrected for medium dielectric effects), $\mathrm{k}$ is the Boltzmann constant, $\mathrm{T}$ is the Kelvin poling temperature, $\mathrm{L}$ is the Langevin function, and $\mathrm{W}$ is the intermolecular electrostatic interaction energy. In this approximation, electronic intermolecular electrostatic (e.g., dipole-dipole) interactions act to reduce poling-induced order realized in the independent particle limit $\mu \mathrm{F} / 5 \mathrm{kT}$. As intermolecular electrostatic interactions increase in a quadratic manner with chromophore number density, a maximum in the graph of electro-optic activity versus number density is predicted with the position of the maximum dependent on chromophore dipole moment. The treatment of intermolecular electrostatic interactions was quickly extended to include treatment of nuclear repulsive interactions at various levels leading to quantitative simulation of the experimental variation of electron-optic activity with chromophore number density for prolate-ellipsoid-shaped chromophores dissolved in polymers to form composite materials. ${ }^{59-62}$ Monte Carlo calculations have also been adapted to the study of intermolecular electrostatic interactions ${ }^{60}$ and recent calculations by Robinson and coworkers ${ }^{62}$ have predicted that intermolecular electrostatic interactions can also lead enhancement of poling-induced acentric order. A variety of effects contribute to the propensity for acentric self-assembly but the essential features can be realized from a consideration of Fig. 1 (derived from Monte Carlo calculations) showing the variation of $\mathrm{N}<\cos ^{3} \theta>$ or Loading Parameter as a function of

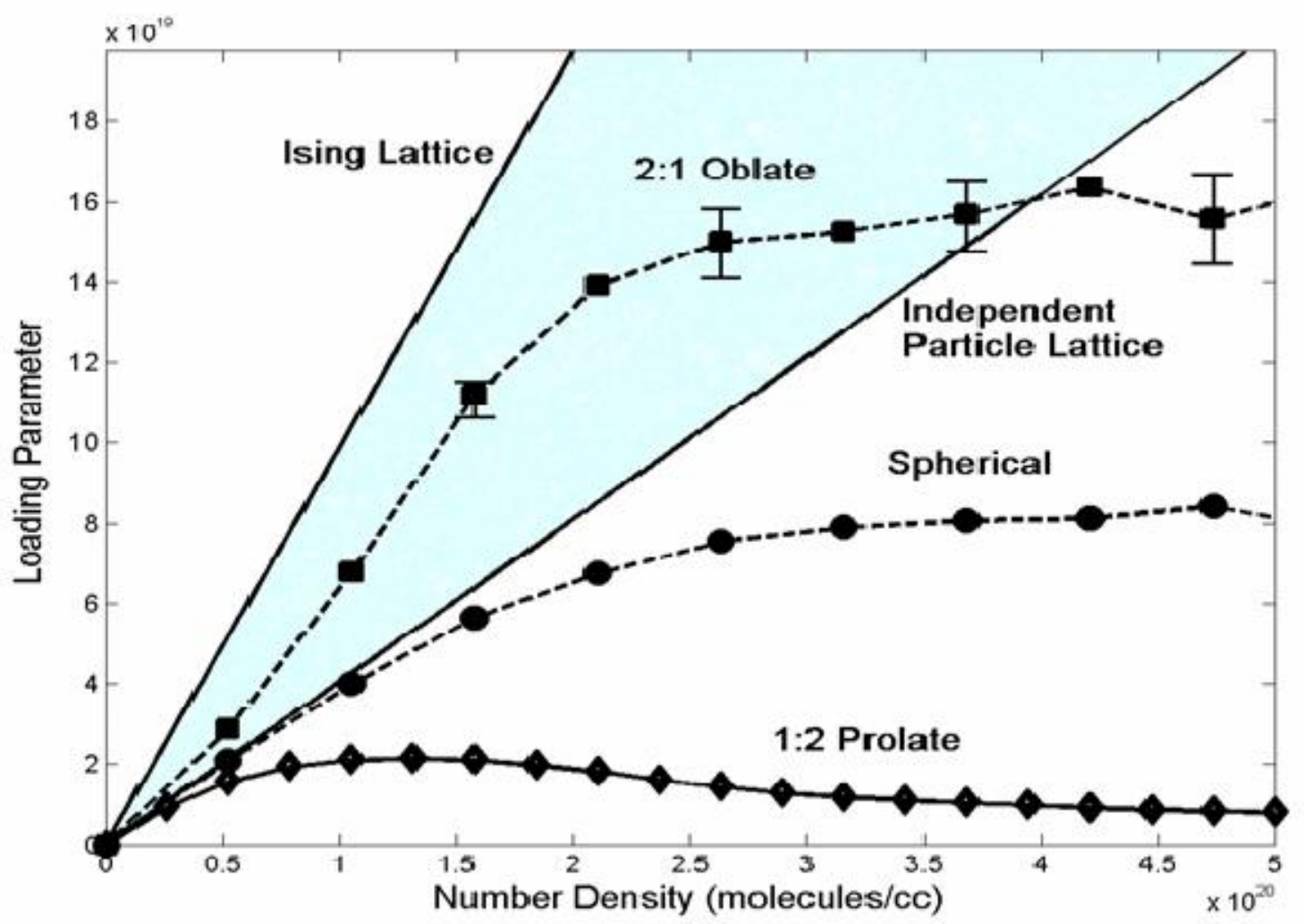

Figure 1. Dependence of electro-optic activity on chromophore shape and number density is shown. Multiplication of the Loading Parameter by molecular first hyperpolarizability, $\beta$, and a constant dependent on medium dielectric yields $\mathrm{r}_{33}$.

N. Plotting $\mathrm{N}<\cos ^{3} \theta>$ rather than $\mathrm{r}_{33}$ versus number density, $\mathrm{N}$, in Fig. 1 has the advantage of providing a more universal curve for discussing the effects of intermolecular electrostatic interactions. In Fig. 1, two limiting cases are 
shown. The independent particle (non-interacting chromophores) case yields an order parameter given by $\left\langle\cos ^{3} \theta>=\right.$ $\mu \mathrm{F} / 5 \mathrm{kT}$ that is independent of chromophore number density or concentration. In this case, the chromophore can access all orientations. The second limiting case is the Ising lattice, where chromophores can only point along the poling field direction or opposite to the poling field direction. The Ising lattice potential is a highly restrictive potential. For the case of an Ising lattice, the order parameter is given by $\left\langle\cos ^{3} \theta>=\mu \mathrm{F} / \mathrm{kT}\right.$ and is a factor of 5 greater than for a chromophore experiencing no confining potential. The Ising model is independent of chromophore number density and thus is of little physical significance other than to set a conceptual limit to the realization of acentric order. In Fig. 1 the area between the independent particle and Ising lattice straight lines represents a region where intermolecular electrostatic interactions act to increase poling-induced acentric order. Below the independent particle line, intermolecular electrostatic interactions act to attenuate poling-induced acentric order. The dependence of curves of electro-optic activity versus number density on chromophore shape can be understood when it is realized that the intermolecular electronic (dipoledipole) electrostatic interaction is spatially anisotropic with one component favoring centrosymmetric ordering of chromophores and with the other component favoring acentric chromophore order. The relative importance of these two contributions changes with chromophore shape. That is, spatially-anisotropic nuclear repulsive interactions for oblateellipsoid-shaped chromophores act to inhibit close side-by-side chromophore approach that favors centrosymmetric order. Such materials prefer discotic head-to-tail stacking that favors acentric or noncentrosymmetric order. From Fig. 1 , it is clear that the functional form of the behavior of $r_{33}$ versus $\mathrm{N}$ changes in going from the region where intermolecular electrostatic interactions oppose poling-induced order to a region where such interactions augment poling-induced order. For oblate-shaped chromophores, the electro-optic activity increases rapidly as a function of $\mathrm{N}$ for low loading and then reaches a saturation value that remains relatively constant with increasing concentration. This is in contrast to the behavior predicted for prolate-ellipsoid-shaped chromophores where the behavior of $\mathrm{r}_{33}$ versus $\mathrm{N}$ follows the independent particle line at low concentrations and then deviates from that line going through a maximum value and decreasing with increasing chromophore number density at high loading. Other potential functions (e.g., those associated with covalent bonds) can also influence the competition of acentric versus centric ordering forces and the functional behavior shown in Fig. 1 provides a useful diagnostic for such competition.

Another important diagnostic for determining whether intermolecular electronic electrostatic interactions augment or attenuate poling-induced order is the ratio $r_{33} / r_{13}$ (see Fig. 2). This ratio has the advantage of canceling out (to first order) the effect of the dielectric medium. The exact magnitude of the ratio will also depend on the anisotropy of the molecular first hyperpolarizability tensor $(\beta)$. However, the functional dependence of the ratio versus number

$$
\begin{aligned}
& b=\frac{\left\{\beta_{z x x}+\beta_{z y y}\right\}}{\beta_{z z z}} \\
& \frac{r_{33}}{r_{13}}=\frac{\chi_{z z z}}{\chi_{z x x}}=\frac{\left\langle\cos ^{3} \theta\right\rangle+b \frac{3}{2}\left\langle\sin ^{2} \theta \cos \theta\right\rangle}{\frac{1}{2}\left\{\left\langle\sin ^{2} \theta \cos \theta\right\rangle+b\left\{\langle\cos \theta\rangle-\frac{3}{2}\left\langle\sin ^{2} \theta \cos \theta\right\rangle\right\}\right\}}
\end{aligned}
$$$$
\frac{\left\langle\sin ^{2} \theta \cos \theta\right\rangle}{\left\langle\cos ^{3} \theta\right\rangle}=\frac{\langle\cos \theta\rangle}{\left\langle\cos ^{3} \theta\right\rangle}-1=\Theta-1
$$

$$
\frac{r_{33}}{r_{13}}=\frac{2+3 b\{\Theta-1\}}{\Theta-1+b\left\{\Theta-\frac{3}{2}(\Theta-1)\right\}}=\frac{2+3 b\{\Theta-1\}}{\Theta-1+\frac{1}{2} b\{3-\Theta\}}
$$




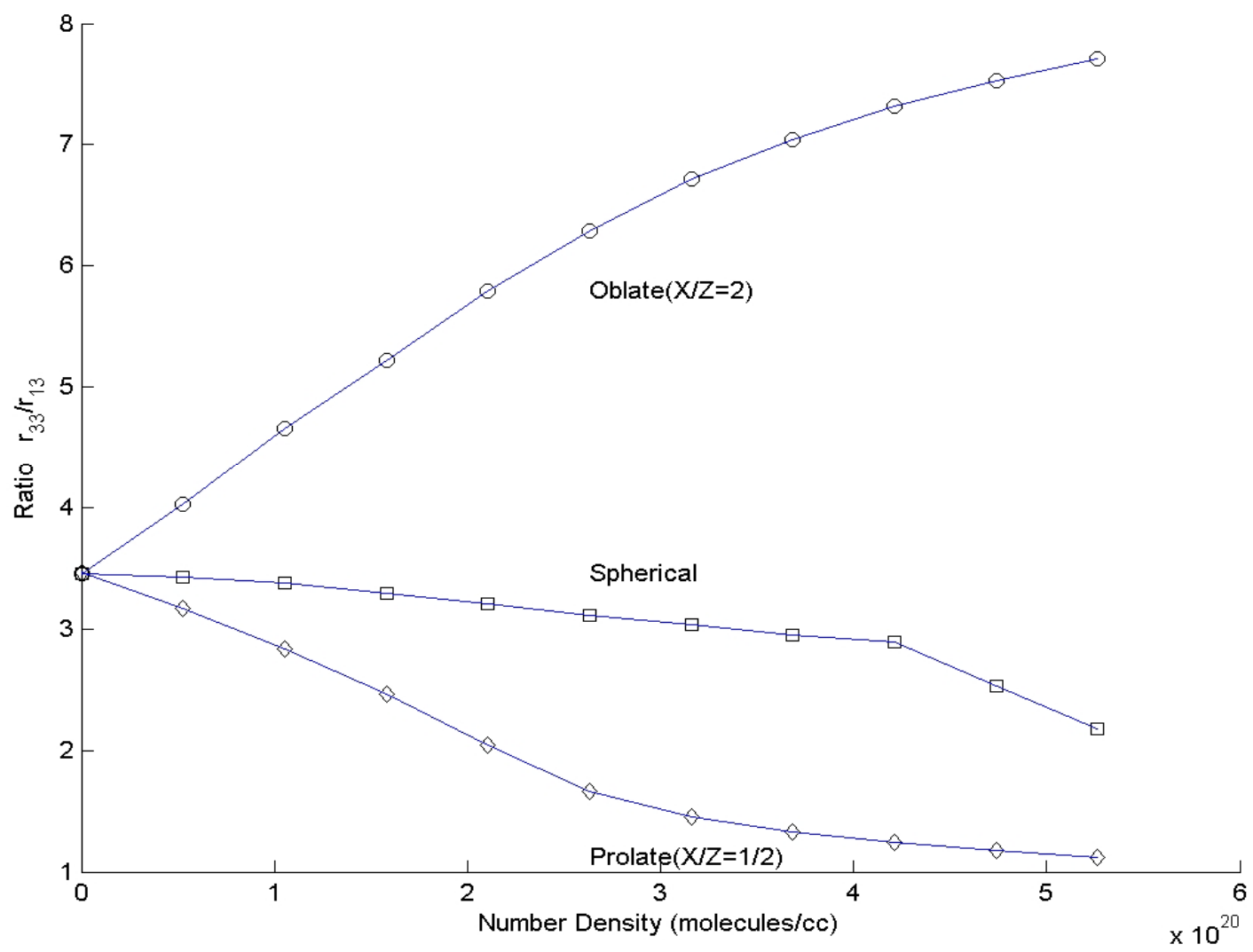

Figure 2. The ratio of $r_{33} / r_{13}$ is shown as a function of chromophore number density for $b=0.2$.

density is diagnostic for the relative importance of various potential functions.

We have also carried out pseudo-atomistic Monte Carlo calculations for multi-chromophore-containing dendrimers as a function of loading in an inert matrix. In the pseudo-atomistic approach, $\pi$-conjugated segments of the dendrimer are treated in a United Atom Approximation while $\sigma$-bonded flexible segments are treated atomistically. We now discuss modeling of a PAS type dendrimer (a three arm dendrimer, one arm of which is shown in Fig. 3.). Pseudoatomistic calculations predict that the acentric order parameter increases with increasing chromophore number density (dendrimer density). Physically, at high concentrations, dendrimers act to restrict the orientations that chromophores can access. This is somewhat analogous to chromophores experiencing an Ising potential. Experimentally, a factor of three improvement in electro-optic activity is observed for the PAS dendrimer over the best values that can be obtained for the same chromophore in a composite material at a concentration that optimizes electro-optic activity. Adding a second class of chromophores to an ordered dendrimer glass may be viewed as adding that chromophore to an Ising-like lattice and enhanced acentric order may follow from such doping to form binary glasses.

In the past year, we have prepared and evaluated a number of new electro-optic materials that can be considered as implementation of the theoretically suggested structures discussed above. A detailed discussion of these dozen materials is beyond the scope of this communication but we attempt to present several examples that illustrate key points. These examples can be categorized as chromophore/multi-chromophore-containing dendrimer and chromophore/single-chromophore-containing dendrimer binary glasses. In Fig. 4, we present data for several representative samples: One doped multi-chromophore-dendrimer system and three different doping levels of doped single-chromophore containing dendrimer material.

Similar results, including even larger electro-optic activities, are observed for other binary doped chromophore glass systems including some systems where electro-optic activity approaches $400 \mathrm{pm} / \mathrm{V}$. In all cases, the electro-optic activities that can be realized are greater than those that can be realized by adding the dopant chromophore to polymers 


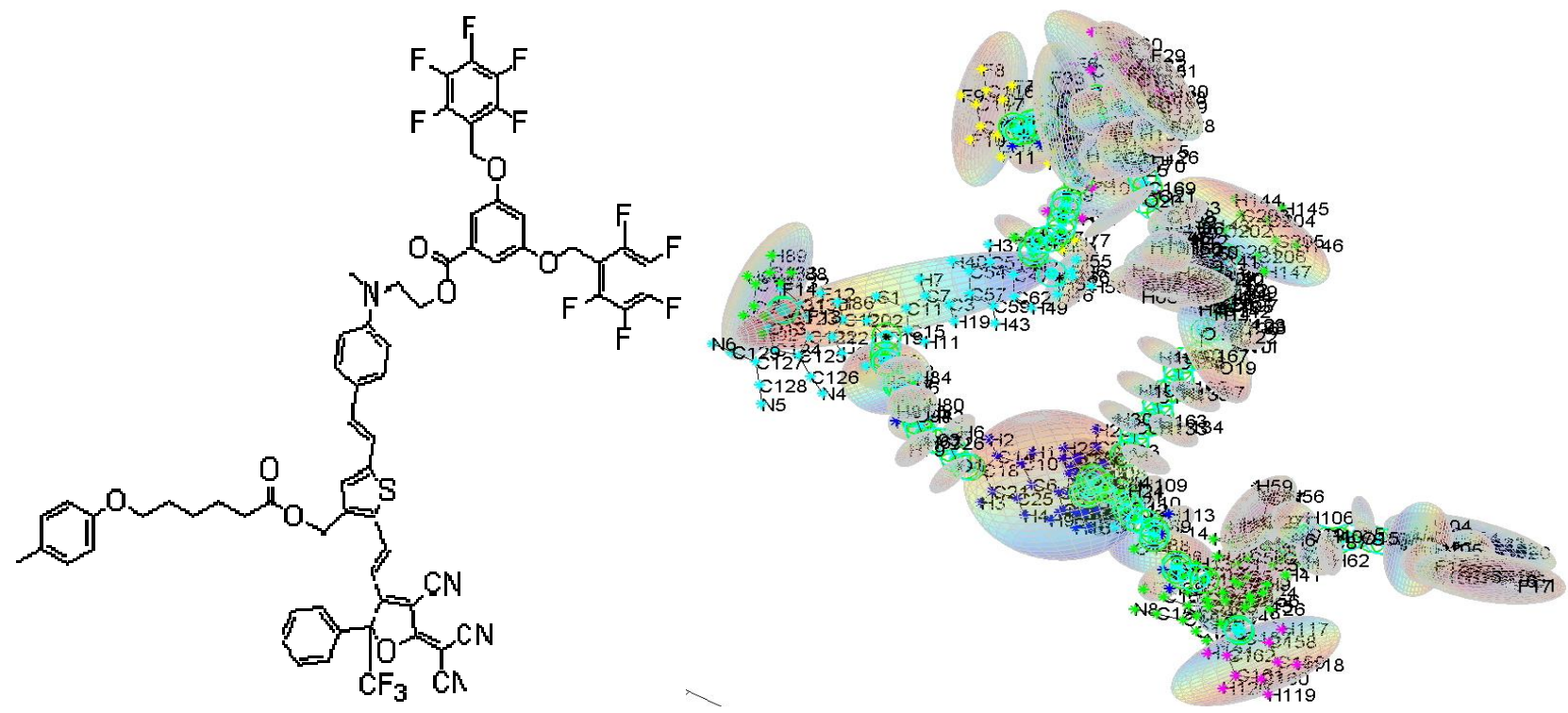

Figure 3. Right. One arm of the three equivalent arms of a PAS type dendrimer is shown. Left. The pseudo-atomistic Monte Carlo picture of the PAS dendrimer is shown.

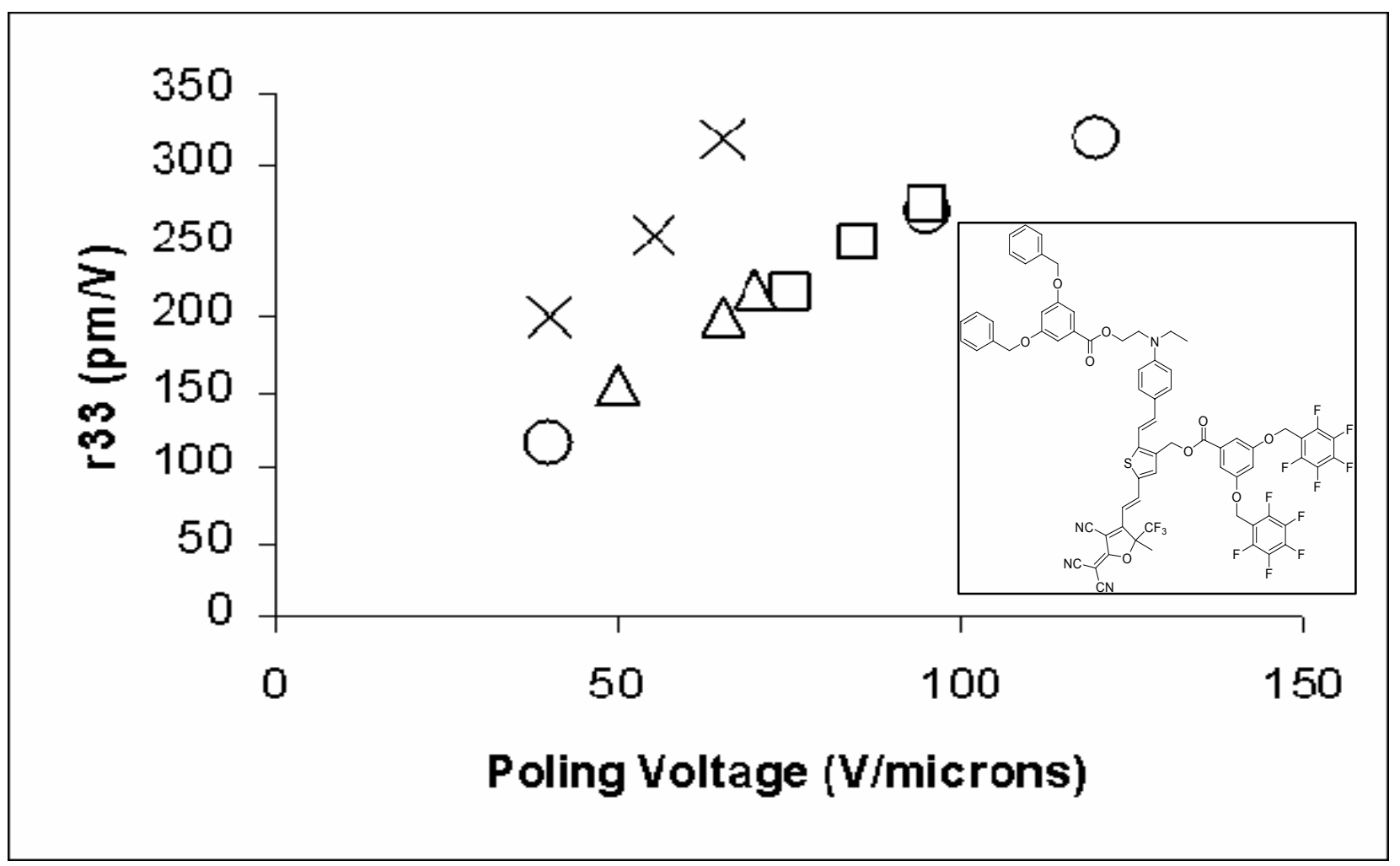

Figure 4. Four data sets for doped binary dendrimer glasses are shown. Circles are for a CLD-like chromophore doped into the PAS dendrimer at an approximately 50\% level. Squares, triangles, and Xs are for different doping levels of a CLD-like chromophore doped into the low generation dendrimer (structure shown in the inset). The molecular first hyperpolarizability of the dopant chromophore is higher than that of the chromophore-containing dendrimers. The behavior of $\mathrm{r}_{33} \mathrm{vs}$. $\mathrm{N}$ for doping is functionally like the behavior for the oblate ellipsoid of Fig. 1. 
such as amorphous polycarbonate. The results are not restricted to the type of chromophore structure considered here and indeed appear to be quite general as long as the dopant and host chromophores are somewhat incommensurate. Our analysis of theoretical and experimental results have suggested a number of experiments for gaining improved information regarding detailed nanoscopic order including measurement of the ratio of $r_{33} / r_{13}$ and nanoscopic imaging based on second harmonic generation, two-photon fluorescence, and birefringence. Theoretical simulations have done a respectable job of qualitatively explaining the experimentally observed trends such as the variation of electro-optic activity and the ratio of $r_{33} / r_{13}$ with chromophore concentration (number density); however, Van der Waals interactions other than dipole-dipole interactions are very strong and ubiquitous suggesting that care be exercised in the analysis of experimental data. The spatially anisotropic electronic dipole-dipole and nuclear repulsive (shape dependent) interactions and potential functions for rotation about covalent bonds are the best understood parts of the problem at this point in time. The critical factor driving self-assembly in the favor of acentric chromophore ordering is the interplay of nuclear repulsive, bond restriction, and Van der Waals interactions to favor the acentric order potential of the electronic dipole-dipole interaction over the centric order favoring component. This is trivially visualized in Fig. 1 when nuclear repulsive potentials are the only interactions beyond electronic dipole-dipole interactions that need to be considered. Fortunately, pseudo-atomistic Monte Carlo calculations provide "snap shot" pictures of various minimum energy structures for the ensemble of chromophores and dendrimers considered. Such snap shots can provide some insight into how chromophores and dendrimers are organizing under the influence of a poling field although one is dealing with Boltzmann aggregates rather than highly organized structures as in the case of single crystalline materials.

Regardless of the precise nanoscopic organization of specific binary glasses, one point is clear from both experimental data and theoretical simulations; namely, that very high chromophore loading and a large acentric order parameter are not mutually exclusive. Indeed, the order parameters and number densities realized for binary organic glasses are beginning to approach those realized for organic crystals. Other properties such as photostability also suggest that binary glasses are closer to organic crystals than chromophore/polymer composites; we have observed photostability to be more than an order of magnitude greater in binary glasses than in chromophore/polymer composites. The observations reported here suggest several things pertinent to the realization of the ultimate electro-optic material. First, it is likely that less than a factor of two additional improvement in electro-optic activity can be realized by further improving the product $\mathrm{N}<\cos ^{3} \theta>$. Further improvement of this product could be viewed as preparing "crystalline" electro-optic materials in a thin film format by solution processing and electric field initiated crystallization. The simple observation is that the easiest route to realizing electro-optic activity of $500 \mathrm{pm} / \mathrm{V}$ or greater is through the incorporation of chromophores of higher $\beta$ values into theoretically-inspired nanostructured lattices such as described in this communication. Another important observation is that chromophore concentrations are much higher in binary glass materials than in chromophore/polymer composite materials and the consequences for optical loss must be considered. Proton densities are typically lower in the doped dendrimer binary glasses so absorption loss due to overtone vibrations of hydrogen will be smaller; however, contributions from chromophore interband (charge transfer) absorption can be higher due to the higher chromophore concentrations. For the types of chromophores considered here, this is unlikely to be a problem at 1.55 microns telecommunications wavelength but care must be exercised in making sure that desired optical loss levels apply at 1.3 microns. Several other observations need to be made with respect to binary dendrimer glasses. These are relatively low molecular weight materials, at least with respect to a high molecular weight polymers such as amorphous polycarbonate. Without crosslinking the glass transition temperatures of the binary glasses of Fig. 4 are in the range $70-90^{\circ} \mathrm{C}$, which is too low for these materials to be useful for the fabrication of practical devices. Fortunately, glass transition temperature can be increased without significantly altering electro-optic activity or optical loss by use of reversible (retro-Diels-Alder) and irreversible (fluorovinyl ether) crosslinking reactions. In studies of other materials, such crosslinking chemistries have been used to improve glass transition temperatures to on the order of $200^{\circ} \mathrm{C}^{63-68}$ In preliminary studies, we have used reversible Diels-Alder crosslinking to increase glass transition temperatures to between $125-150^{\circ} \mathrm{C}$ for the materials of Fig. 4. Achieving the highest desired glass transition temperatures $\left(\sim 200^{\circ} \mathrm{C}\right)$ without attenuation of electro-optic activity or increase in optical loss requires attention to both the segmental flexibility of crosslinked moieties and the positioning of crosslinking functionalities. While the highest glass transition temperatures are desirable for the greatest thermal and photochemical stability, lower values can be important for processing protocols such as soft and nanoimprint lithography.

The binary glass materials, described in this communication, exhibit record fast-response electro-optic activity. Providing that the glass transition temperatures of the materials can be increased to $150-200^{\circ} \mathrm{C}$ and providing that low optical loss waveguides can be fabricated from the materials, these materials should be useful for fabricating a wide array of ultra high bandwidth, low drive voltage devices. Preliminary success has already been realized in incorporating these materials into silicon photonic split ring microresonator devices where organic electro-optic materials may not only 
be useful for electrical-to-optical signal transduction and optical switching but also for high bandwidth optical rectification. ${ }^{20}$ These materials may be useful for terahertz signal generation and detection providing both exceptional sensitivity and high bandwidth. ${ }^{12,13}$ The absence of phonon modes associated with crystalline materials affords greater bandwidth in the $1-20 \mathrm{THz}$ range. ${ }^{12,13}$

\section{Fabrication of Ring Microresonators}

There is not sufficient space to describe research on both all-organic and organic OEO/silicon photonic hybrid ring microresonators and the theory behind device design. The reader is referred to recent publications , $^{97-21,36-46}$ for a broader picture including to the fabrication of all-organic device structures by more novel techniques such as nanoimprint ${ }^{17-21}$ and two-photon ${ }^{42,44}$ lithography. Here we focus on presenting two different approaches to the fabrication of hybrid devices where the low dielectric breakdown threshold of silicon provides a challenge to induction of electro-optic activity by electric field poling.

The high index of silicon provides a natural platform for concentrating or tightly confining light in circuits of nano/microscopic dimensions. ${ }^{68-72}$ Moreover, it has recently been shown that it is possible to concentrate optical power in the slot of slotted silicon photonic devices..$^{20,73,74}$ providing a low loss transition from high index silicon to lower index of refraction OEO materials. The high Qs of such devices permit an amplification of optical field intensities that results in optical rectification. ${ }^{20}$ Thus, the potential exists for using the same slotted ring microresonator device structure for both electrical-to-optical and optical-to-electrical signal transduction on the same chip.

We have fabricated prototype device structures using CLD/APC composite materials, ${ }^{31}$ CLD composites with the chromophore (YLD124) of Fig. 5, and the binary glasses discussed in this communication. Progression through

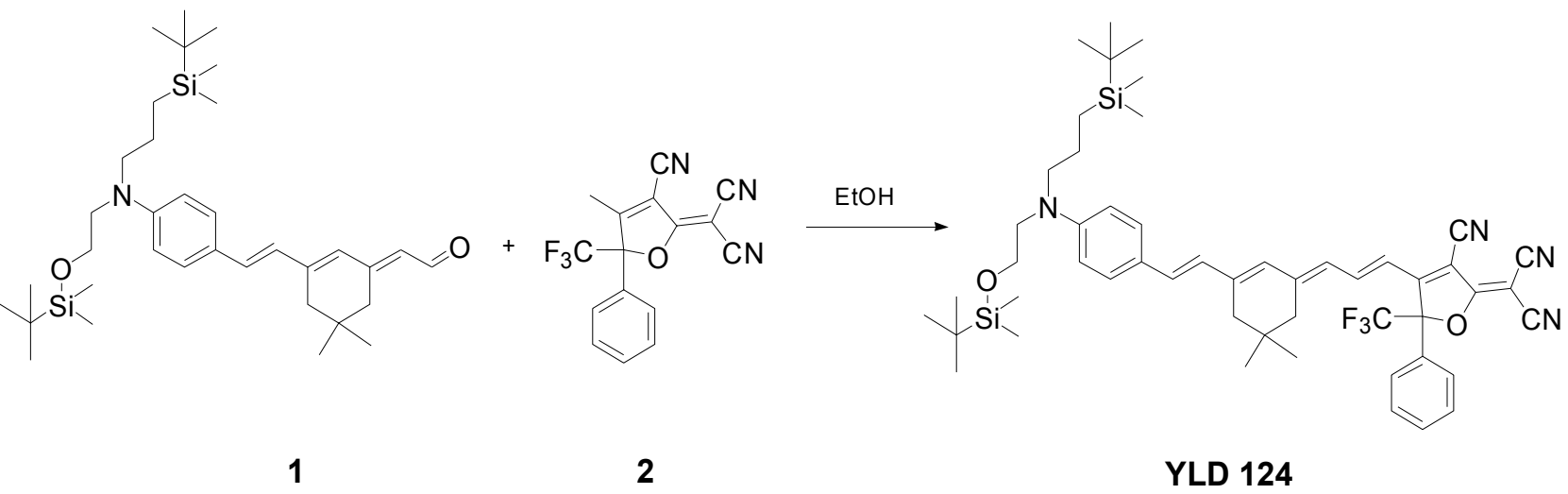

Fig. 5. The structure of the YLD 124 chromophore is shown.

these materials has led to dramatic improvements in device performance parameters such as the bandwidth/voltage (sensitivity) factor. The YLD124 chromophore can be considered a variant of the CLD chromophore where the two methyl groups of the acceptor furan ring are replaced by a trifluoromethyl group and a phenyl group. This modification leads to an approximate factor of two improvement in chromophore $\beta$ value. ${ }^{56}$ We provide examples of use of the first two materials here. Publication of the results on binary glasses and dendrimer glasses will be presented elsewhere.

Several different device structures have been explored including the two structures discussed in this communication. Both of the devices discussed here are based on depositing OEO materials by spin casting. Other methods of deposition, including vapor phase deposition, have been successfully explored. In the first device structure, shown in Fig. 6, the CLD/APC material is used; electric field poling and drive voltages are provided by metal electrode structures. Typical results, showing both voltage (electro-optic) and thermal wavelength tuning are shown in Fig. 7. As is evident from consideration of these results, the electro-optic activity of the OEO material is considerably attenuated from that typically realized in all-organic devices due to the dielectric breakdown limitations of silicon. For that reason, we have explored the use of conducting silicon electrodes (Electronic grade silicon was implanted with phosphorous at normal incidence, $30 \mathrm{keV}$ energy, and $1 \times 10^{12}$ ions $/ \mathrm{cm}^{2}$ density). The device structure and typical results are shown in 


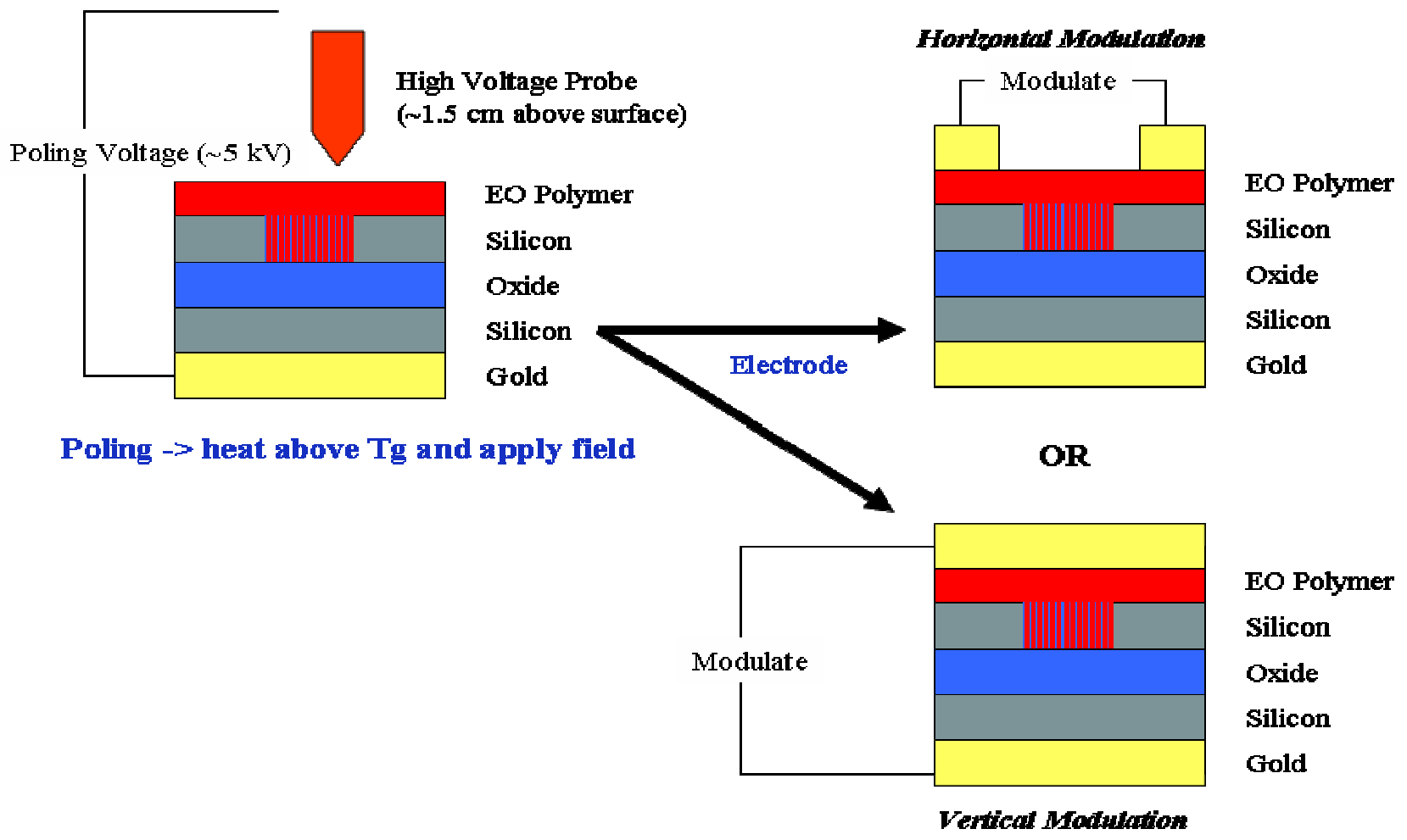

Figure 6. Preparation of $\mathrm{OEO} /$ silicon photonic hybrid ring microresonator devices by corona poling is shown. Both horizontal and vertical modulation have been explored.
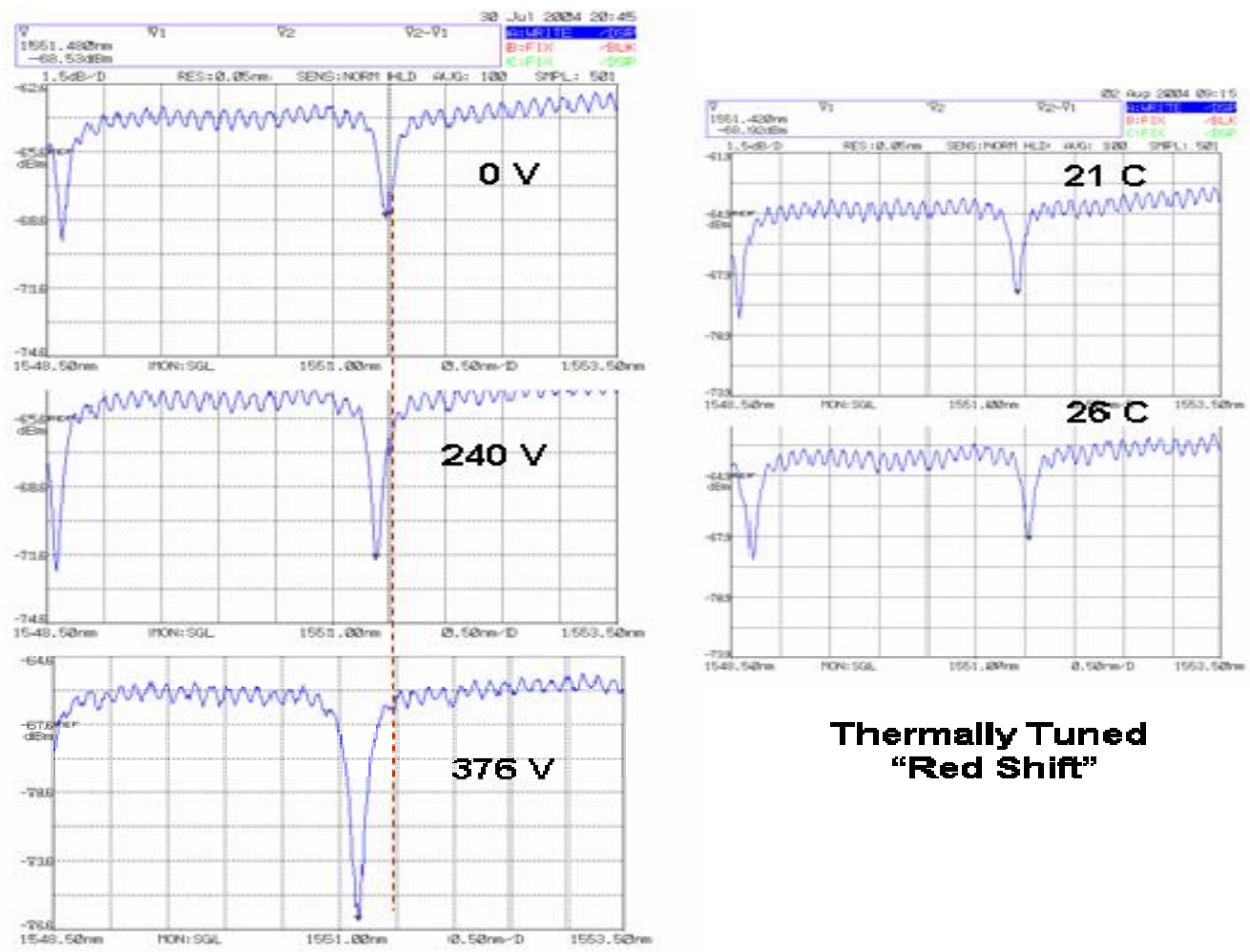

Thermally Tuned

"Red Shift"

Figure 7. Voltage (above left) and thermal (above right) tuning of the devices of Fig. 6 are shown. 
Fig. 8. More details on fabrication are given in reference 20. For such devices, bandwidth/voltage factors on the order

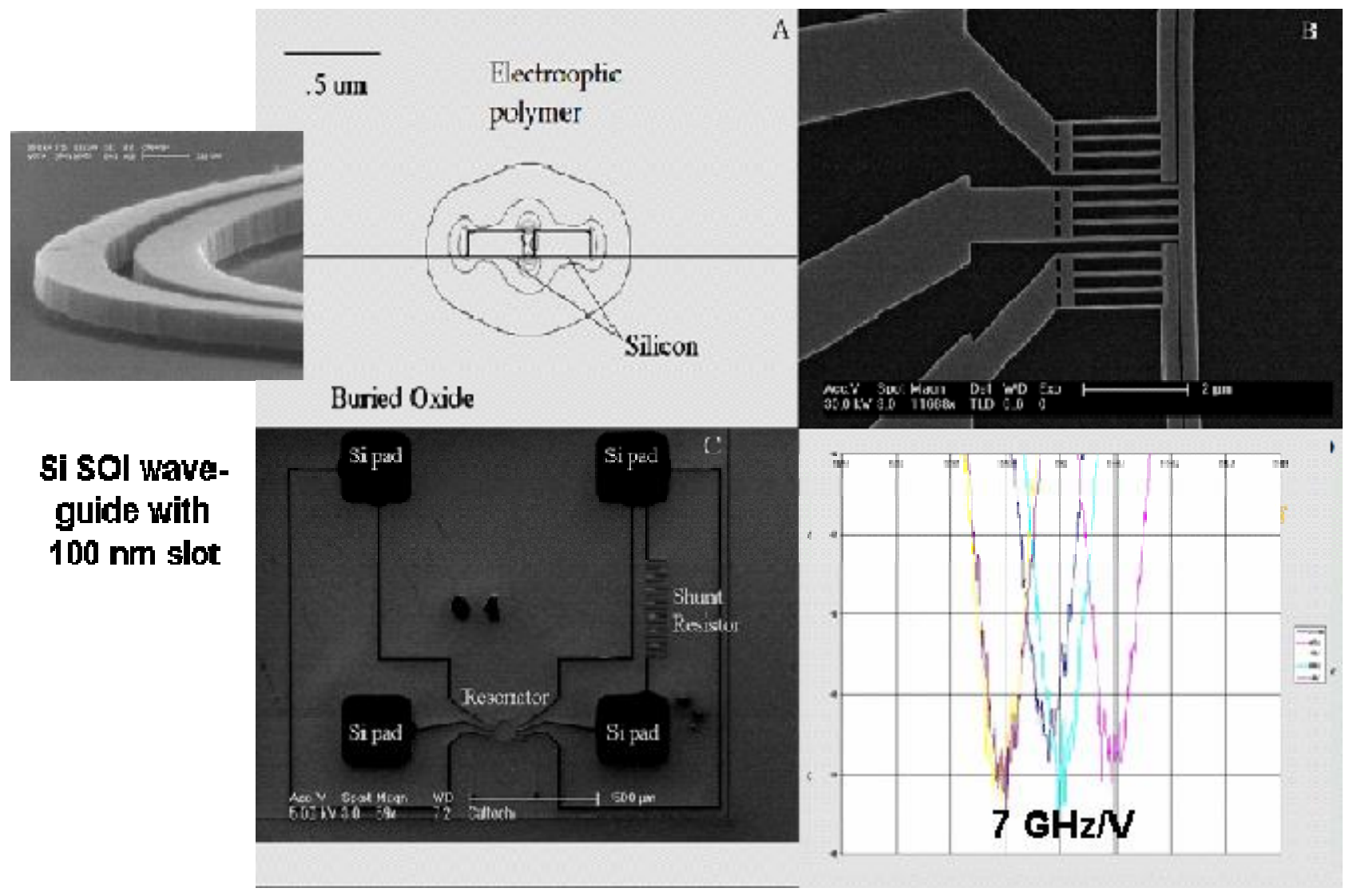

Figure 8. The structure and performance of an OEO/silicon photonic slotted ring microresonator are shown. Slot widths ranging from $70-120 \mathrm{~nm}$ have been examined.

of $7 \mathrm{GHz} / \mathrm{V}$ have been observed. Various poling experiments demonstrated that effective electro-optic coefficients for the YLD124/APC composite material of $80-100 \mathrm{pm} / \mathrm{V}$ could be realized with conducting silicon electrodes due to the close electrode spacing. These values are greater than those obtained in conventional poling of all-organic EO structures.

\section{Summary}

Statistical mechanical calculations have been used to understand the improvement of the electro-optic activity of organic materials to values greater than $300 \mathrm{pm} / \mathrm{V}$. New binary glasses demonstrate that intermolecular electrostatic interactions can enhance (as well as detract from) poling-induced acentric order. Dramatically improved values of the product $\mathrm{N}<\cos ^{3} \theta>$ have been obtained for binary glasses of chromophores and chromophore-containing dendrimers. Such binary glasses can be viewed as approaching crystalline organic electro-optic materials in terms of chromophore number density and acentric order parameter. The greatest chance for further dramatic improvement in electro-optic activity for organic materials appears to involve improving the molecular hyperpolarizability of constituent chromophores. Quantum mechanical calculations suggest that significant further improvement can be obtained by simple modification of donor, bridge, and acceptor moieties of charge-transfer chromophores. Certainly, values in the range 500-1000 pm/V appear obtainable in the near future exploiting quantum mechanical guidance. Of course, for new materials to be of utility for the fabrication of devices, they must exhibit adequate stability. Reversible (Retro-DielsAlder) and irreversible (fluorovinyl ether crosslinking to form cyclobutyl bridges) crosslinking chemistries afford the opportunity to dramatically increase stability without compromise of electro-optic activity or optical loss. OEO materials have been used to fabricate both all-organic and OEO/silicon photonic hybrid ring microresonator devices. 
Bandwidth/voltage sensitivity factors in the range 1-10 GHz/V are observed for devices fabricated using CLD-type chromophore/APC polymer composite materials. Values greater than $30 \mathrm{GHz} / \mathrm{V}$ can be anticipated with use of new binary glass $\mathrm{OEO}$ materials.

\section{Acknowledgments}

The authors wish to thank the Air Force Office of Scientific Research and the National Science Foundation (DMR-0092380 and DMR-0120967) for partial support of this research.

\section{References}

1. Polymers for Lightwave and Integrated Optics, edited by L. A. Hornak, Marcel Dekker, New York, 1992.

2. Nonlinear Optics of Organic Molecules and Polymers, edited by H. S. Nalwa, S. Miyata, CRC Press, Boca Raton 1997.

3. Electrical and Optical Polymer Systems: Fundamentals, Methods and Applications, edited by D Wise, G. Wnek, D. Trantolo, T. Cooper, J. Gresser, Marcel Dekker, New York, NY, 1998.

4. Characterization Techniques and Tabulations for Organic Nonlinear Optical Materials, edited by M. G. Kuzyk and C. W. Dirk, Marcel Dekker, New York, 1998.

5. Polymers for Lightwave and Integrated Optics, edited by L. A. Hornak, Marcel Dekker, New York, 1992.

6. L. R. Dalton, A. W. Harper, A. Ren, F. Wang, G. Todorova, J. Chen, C. Zhang, and M. Lee, Polymeric electrooptic modulators: From chromophore design to integration with semiconductor VLSI electronics and silica fiber optics, Ind. Eng. Chem. Res. 38, pp. 8-33 (1999).

7. L. R. Dalton, "Nonlinear Optical Polymeric Materials: From Chromophore Design to Commercial Applications," in Advances in Polymer Science, Vol. 158, edited by K. S. Lee, Springer, Heidelberg, pp. 1-86, 2002.

8. L. R. Dalton, "Rational Design of Organic Electro-Optic Materials," J. Phys.: Condens. Matter, 15, pp. R897-R934 (2003).

9. L. R. Dalton, "Organic Electro-Optic Materials," in Handbook of Conducting Polymers, edited by T. Skotheim and J. Reynolds, CRC Press/Marcel Dekkar, Boca Raton, 2006, submitted.

10. D. Chen, H. R. Fetterman, A. Chen, W. H. Steier, L. R. Dalton, W. Wang, and Y. Shi, "Demonstration of $110 \mathrm{GHz}$ Electro-Optic Polymer Modulators," Appl. Phys. Lett., 79, pp. 3335-3337 (1997).

11. M. Lee, H. E. Katz, C. Erben, D. M. Gill, P. Gopalan, J. D. Heber, and D. J. McGee, " Ultra-fast modulation of light using an electro-optic polymer," Science, 289, pp. 1404-1407 (2002).

12. A. M. Sinyukov and L. Michael Hayden, "Efficient Electrooptic Polymers for THz Applications," J. Phys. Chem. $B$, 108, pp. 8515-8522 (2004).

13. A. M. Sinyukov, M. R. Leahy, L. M. Hayden, M. Haller, J. Luo, A. K. Y. Jen, and L. R. Dalton, "Resonance Enhanced THz Generation in Electro-Optic Polymers Near the Absorption Maximum" Applied Physics Letters, 85, pp. 5827-5829 (2004).

14. P. Zhu, H. Kang, M. E. van der Boom, Z. Liu, G. Xu, J. Ma, D. Zhou, S. T. Ho, and T. J. Marks, "Self-assembled materials and devices that process light," Proc. SPIE, 5621, pp. 105-116 (2004).

15. J. Li, P. J. Neyman, M. Vercellino, J. R. Heflin, R. Duncan, and S. Evoy, "Active Photonic Crystal Devices in SelfAssembled Electro-Optic Polymeric Materials," Mat. Res. Soc. Symp. Proc. 817, pp. 133-138 (2004).

16. H.-C. Song, M.-C. Oh, S.-W. Ahn, W. H. Steier, H. R. Fetterman, and C. Zhang, "Flexible, Low-Voltage ElectroOptic Polymer Modulators," Applied Physics Letters, 82, pp. 4432-4434 (2003).

17. Y. Huang, G. T. Paloczi, A. Yariv, C. Zhang, and L. R. Dalton, "Fabrication and Replication of Polymer Integrated Optical Devices Using Electron-Beam Lithography and Soft Lithography," Journal of Physical Chemistry, 108, pp. 8006-8613 (2004).

18. G. T. Paloczi, Y. Huang, A. Yariv, J. Luo, A. Jen, "Replica-Molded Electro-Optic Polymer Mach-Zehnder Modulator," Applied Physics Letters, 85, pp. 1662-1664 (2004).

19. K. A. Firestone, P. Reid, R. Lawson, S. H. Jang, and L. R. Dalton, "Advances in Organic Electro-Optic Materials and Processing," Inorg. Chem. Acta 357, pp. 3957-3966 (2004).

20. T. Baehr-Jones, M. Hochberg, G. Wang, R. Lawson, Y. Liao, P. A. Sullivan, L. R. Dalton, A. K. Y. Jen, and A. Scherer, "Optical Modulation and Detection in Slotted Silicon Waveguides," Optics Express, 13, pp. 5216-5226 (2005).

21. B. Maune, R. Lawson, C. Gunn, A. Scherer, and L. Dalton, "Electrically Tunable Ring Resonators Incorporating Nematic Liquid Crystals as Cladding Layers," Appl. Phys. Lett. 83, pp. 4689-4691 (2003). 
22. A. Chen, V. Chuyanov, F. I. Marti-Carrera, S. M. Garner, W. H. Steier, J. Chen, S. S. Sun, L. R. Dalton, "Vertically Tapered Polymer Waveguide Mode Size Transformer for Improved Fiber Coupling," Opt. Eng., 39, pp. 1507-1516 (2000).

23. S. M. Garner, S. S. Lee, V. Chuyanov, A. Chen, A. Yacoubian, W. H. Steier, and L. R. Dalton, "Three Dimensional Integrated Optics Using Polymers," IEEE J. of Quantum Electronics, 35, pp. 1146-1155 (1999).

24. W. Steier, A. Chen, S. Lee, S. Garner, H. Zhang, V. Chuyanov, L. Dalton, F. Wang, A. Ren, C. Zhang, G. Todorova, A. Harper, H. Fetterman, D. Chen, A. Udupa, D. Bhattacharya, and B. Tsap, "Polymer Electro-Optic Devices for Integrated Optics," Chem.Phys.,245, pp. 487-506 (1999).

25. A. Chen, V. Chuyanov, F. I. Marti-Carrera, S. Garner, W. H. Steier, J. Chen, S. Sun, and L. R. Dalton, "Integrated Polymer Waveguide Mode Size Transformer with a Vertical Taper for Improved Fiber Coupling," Proc. SPIE 3005, 65-76 (1997).

26. M. C. Oh, C. Zhang, H. J. Lee, W. H. Steier, and H. R. Fetterman, "Low-Loss Interconnection Between Electrooptic and Passive Polymer Waveguides With a Vertical Taper," IEEE Phot. Tech. Lett. 14, 1121-1123 (2002).

27. D. Y. Zang, G. Shu, T. Downing, W. Lin, C. Oh, and J. Bechtel, "Insertion loss reduction in high speed polymer electrooptic modulators using tapered waveguide, fiber tip lenses and modification of waveguide structures," Proc. SPIE 4991, 601-609 (2003).

28. D. H. Chang, T. Azfar, S. K. Kim, H. R. Fetterman, C. Zhang, and W. H. Steier, "Vertical adiabatic transition between silica planar waveguide and electro-optic polymer fabricated using grayscale lithography," Optics Lett. 28, 869-871 (2003).

29. Y. Enami, G. Meredith, N. Peyghambarian, M. Kawazu, and A. K. Y. Jen, "Hybrid electro-optic polymer and selectively buried sol-gel waveguides," Appl. Phys. Lett. 82, 490-492 (2003).

30. S. E. Soggs, A. Chen, L. R. Dalton, and B. Parviz, "Integration of Electro-Optic Polymer Waveguide Devices with n-Channel FET Control Circuitry on Silicon," Proc. SPIE, 5730, pp. 125-132 (2005).

31. Y. Shi, C. Zhang, H. Zhang, J. H. Bechtel, L. R. Dalton, B. H. Robinson, and W. H. Steier, "Low (Sub-1 Volt) Halfwave Voltage Polymeric Electrooptic Modulators Achieved by Control of Chromophore Shape," Science, 288, pp. 119-122 (2000).

32. J. H. Bechtel, J. Menders, and D. Y. Zang, "Electro-optic Polymer Integrated Optic Devices and Future Applications," Proc. SPIE, 4991, pp. 552-561 (2003).

33. J. Han, B. J. Seo, H. R. Fetterman, H. Zhang, and C. Zhang, "New Applications Using Single-Sideband Polymer Electro-Optic Modulators," Proc. SPIE, 4991, pp. 562-574 (2003).

34. C. H. Cox III and E. I. Ackerman, "High Electro-Optic Sensitivity (r33) Polymers: They Are Not Just for Low Voltage Modulators Any More," J. Phys. Chem. B, 108, pp. 8540-8542 (2004).

35. L. Sun, J. Kim, C. Jang, D. An, X. Lu, Q. Zhou, J. M. Taboada, R. T. Chen, J. J. Maki, S. Tang, H. Zhang, W. H. Steier, C. Zhang, and L. R. Dalton, "Polymeric Waveguide Prism Based Electro-Optic Beam Deflector," Opt. Eng., 40, pp. 1217-1222 (2001).

36. P. Rabiei, W. H. Steier, C. Zhang, and L. R. Dalton, "Polymer Micro-Ring Filters and Modulators," J. Lightwave Technology, 20, pp. 1968-75 (2002).

37. P. Rabiei, W. H. Steier, C. Zhang, and L. R. Dalton, "Micro-Photonic Polymer Device," International Optical Communications, pp. 14-16 (Autumn, 2002).

38. P. Rabiei, W. H. Steier, C. Zhang, and L. R. Dalton, "Integrated WDM Polymer Modulator," in Optical Society of America Trends in Optics and Photonics, Vol. 70, Optical Fiber Communication Conference, Technical Digest (Optical Society of America, Washington DC, 2002), pp. 31-33.

39. P. Rabiei and W. H. Steier, "Tunable Polymer Double Micro-ring (DMR) Filters," IEEE Phot. Tech. Lett. 15, pp. 1255-1257 (2003).

40. L. R. Dalton, B. H. Robinson, R. Nielsen, A. K. Y. Jen, P. Rabiei, and W. H. Steier, "Organic Electro-Optics: Exploiting the Best of Electronics and Photonics," Proc. SPIE, 4991, pp. 508-519 (2003).

41. L. Dalton, A. Jen, W. Steier, B. Robinson, S. H. Jang, O. Clot, H. C. Song, Y. H. Kuo, C. Zhang, P. Rabiei, S. W. Ahn, and M. C. Oh, "Organic Electro-Optic Materials: Some Unique Opportunities," Proc. SPIE, 5351, pp. 1-15 (2004).

42. T. J. Sherwood, A. C. Young, J. Takayesu, A. K. Jen, L. R. Dalton, and A. Chen, "Polymer Ring Resonator Made by Two-Photon Polymerization and Vertically Coupled to a Side-Polished Optical Fiber," Proc. SPIE, 5724, pp. 356-366 (2005). 
43. A. Pyajt, L. Dalton, and A. Chen, "Novel wavelength selective switch based on electro-optic polymer microrings," Proc. SPIE 5935, in press (2005).

44. T. Sherwood, A. C. Young, J. Takayesu, A. K.Y. Jen, L. R. Dalton, and A. Chen, "Microresonator on SidePolished Optical Fiber," Photonics Technology Letters, in press.

45. Y. Huang, J. K. S. Poon, W. Liang, A. Yariv, C. Zhang, and L. R. Dalton, "Combined Electromagnetic and Photoreaction Modeling of CLD-1 Photobleaching in Polymer Microring Resonators," Appl. Phys. Lett., submitted.

46. A. Chen, L. R. Dalton, T. J. Sherwood, A. K. Jen. P. Rabiei, W. H. Steier, Y. Huang, G. T. Paloczi, J. K. Poon, A. Scherer, and A. Yariv, "All-Organic and Organic-Silicon Photonic Ring Microresonators", Proc. SPIE, 5708, pp. 187-197 (2005).

47. L. R. Dalton, B. H. Robinson, A. K. Y. Jen, P. Ried, B. Eichinger, S. H. Jang, J. Luo, S. Liu, Y. Liao, K. Firestone, N. Bhatambrekar, D. Bale, M. Haller, S. Bhattacharjee, J. Schendel, P. Sullivan, S. Hammond, N.Buke r, F. Cady, A. Chen, and W. Steier, "Electro-optic coefficients of $500 \mathrm{pm} / \mathrm{V}$ and beyond for organic materials," Proc. SPIE, 5621, pp. 93-105 (2004).

48. L. Dalton, B. Robinson, A. Jen, P. Ried, B. Eichinger, P. Sullivan, A. Akelaitis, D. Bale, M. Haller, J. Luo, S. Liu, Y. Liao, K. Firestone, N. Bhatambrekar, S. Bhattacharjee, J. Sinness, S. Hammond, N. Buker, R. Snoeberger, M. Lingwood, H. Rommel, J. Amend, S. H. Jang, A. Chen, and W. Steier, "Acentric lattice electro-optic materials by rational design," Proc. SPIE, 5912, in press (2005).

49. L. Dalton, B. Robinson, A. Jen, P. Ried, B. Eichinger, P. Sullivan, A. Akelaitis, D. Bale, M. Haller, J. Luo, S. Liu, Y. Liao, K. Firestone, A. Sago, N. Bhatambrekar, S. Bhattacharjee, J. Sinness, S. Hammond, N. Buker, R. Snoeberger, M. Lingwood, H. Rommel, J. Amend, S. H. Jang, A. Chen, and W. Steier, "Optimizing electro-optic activity in chromophore/polymer composites and in organic chromophore glasses," Proc. SPIE, 5990, in press (2005).

50. M. Haller, Ph.D. Thesis, University of Washington, Seattle, WA (2005).

51. Y. Zhou, L. Shaojun, Y. Cheng, "Poling Properties of Guest-Host Polymer Films of X-Type Nonlinear Optical Chromophores," Synth. Met. 147, pp. 1519-1520 (2003).

52. P. A. Sullivan, S. Bhattacharjee, B. E. Eichinger, K. Firestone, B. H. Robinson, and L. R. Dalton, "Exploration of Series Type Multifuctionalized Nonlinear Optical Chromophore Concept," Proc. SPIE, 5351, pp. 253-259 (2004).

53. H. Kang, P. Zhu, Y. Yang, A. Facchetti, and T. J. Marks, "Self-Assembled Electrooptic Thin Films with Remarkably Blue-Shifted Optical Absorption Based on an X-Shaped Chromophore," J. Am. Chem. Soc., 126, pp. 15974-15975 (2004).

54. A. Facchetti, G. R. Hutchison, S. Keinan, and Mark Ratner, "Control mechanisms for transport and nonlinear optical response in organic materials: a tale of twists and barriers," Inorganica Chimica Acta, 357, pp. 3980-3990 ((2004).

55. H. Kang, A. Facchetti, H. Jiang, P. Zhu, and T. J. Marks, "Synthesis and Unprecedented Electro-Optic Response Properties of Twisted $\pi$-System Chromophores," Mat. Res. Soc. Symp. Proc., in press.

56. Y. Liao, B. E. Eichinger, K. A. Firestone, M. Haller, J. Luo, W. Kaminsky, J. B. Benedict, P. J. Reid, A. K-Y Jen, L. R. Dalton, B. H. Robinson, "Systematic Study of the Structure-Property Relationship of a Series of Ferrocenyl Nonlinear Optical Chromophores", Journal of the American Chemical Society, 127, pp. 2758-2766 (2005).

57. R. R. Barto, Jr., P. V. Bedworth, J. Epstein, S. P. Ermer, and R. E. Taylor, "Effects of alkyl spacer group length on Vis-NIR absorption behavior in FTC-like guest host EO polymers," Proc. SPIE 4991, pp. 575-588 (2003).

58. R. R. Barto, Jr., C. W. Frank, P. V. Bedworth, S. Ermer, and R. E. Taylor, "Near-Infrared Optical Absorption Behavior in High-b Nonlinear Optical Chromophore-Polymer Guest-Host Materials. 1. Continuum Dielectric Effects in Polycarbonate Hosts,” J. Phys. Chem. B 108, pp. 8702-8715 (2004).

59. L. R. Dalton, A. W. Harper, and B. H. Robinson, "The Role of London Forces in Defining Noncentrosymmetric Order of High Dipole Moment-High Hyperpolarizability Chromophores in Electrically Poled Polymeric Thin Films," Proc. Natl. Acad. Sci. USA, 94, pp. $4842-4847$ (1997).

60. B. H. Robinson and L. R. Dalton, "Monte Carlo Simulations of the Effect of a Poling Field on the Ordering of High Dipole Moment Organic Chromophores," J. Phys. Chem., 104, pp. 4785-4795 (2000).

61. L. R. Dalton, B. H. Robinson, A. K. Y. Jen, W. H. Steier, and R. Nielsen, "Systematic Development of High Bandwidth, Low Drive Voltage Organic Electro-Optic Devices and Their Applications," Opt. Mater., 21, pp. 19-28 (2003).

62. R. D. Nielsen, H. L. Rommel, and B. H. Robinson, "Simulation of the Loading Parameter in Organic Nonlinear Optical Materials" J. Phys. Chem. B, 108, pp. 8659-8667 (2004). 
63. N. Tucker, H. Li, H. Tang, L. R. Dalton, Y. Liao, B. H. Robinson, A. K. Jen, J. Luo, S. Liu, M. Haller, J. Kang, T. Kim, S. Jang and B. Chen, "Recent Progress in Developing Highly Efficient and Thermally Stable Nonlinear Optical Polymers for Electro-Optics," Proc. SPIE, 5351, pp. 36-43 (2004).

64. M. Haller, J. Luo, Hongxian Li, T. D. Kim, Y. Liao, B. Robinson, L. R. Dalton, and A. K. Y. Jen, "A Novel Lattice-Hardening Process to Achieve Highly Efficient and Thermally Stable Nonlinear Optical Polymers," Macromolecules, 37, pp. 688-690 (2004).

65. S. Suresh, S. Chen, C. M. Topping, J. M. Ballato, and D. W. Smith, Jr., "Novel Perfluorocyclobutyl(PFCB) polymers containing isophorone derived chromophore for electro-optic [EO] applications," Proc. SPIE, 4991, pp. 530-536 (2003).

66. C. Zhang, H. Zhang, M. C. Oh, L. R. Dalton, and W. H. Steier, "What the Ultimate Polymeric Electro-Optic Materials will be: Guest-Host, Crosslinked, or Side-Chain?” Proc. SPIE, 4991, pp. 537-551 (2003).

67. J. Luo, M. Haller, H. Ma, S. Liu, T. D. Kim, Y. Tian, S. H. Jang, B. Chen, L. R. Dalton, and A. K-Y. Jen, "Nanoscale Architectural Control and Macromolecular Engineering of Nonlinear Optical Dendrimers and Polymers for Electro-Optics," J._Phys. Chem. B , 108, pp. 8523-8530 (2004).

68. D. Jin, T. Londergan, D. Huang, N. Wolf, s. Condon, D. Tolstedt, H. W. Guan, S. Cong, E. Johnson, and R. Dinu, "Achieving Large Electro-Optic Response: DH-Type Chromophores In Both Crosslinked Systems and Linear High Tg Systems," Proc. SPIE, 5351, pp. 44-56 (2004).

69. R. A. Soref and J. P. Lorenzo, "All-Silicon Active and Passive Guided-Wave Components for Lambda=1.3 and 1.6MU-M” IEEE J. Quantum Electron., 22, pp. 873-879 (1986).

70. A. Lavadi, A. Vonsovici, R. Orobtchouk, D. Pascal, and A. Koster, "Low-loss optical waveguide on standard SOI/SIMOX substrate," Opt. Commun., 146, 31-33 (1998).

71. A. S. Liu, R. Jones, L. Liao, D. Samara-Rubio, D. Rubin, O. Cohen, R. Nicolaescu, and M. Paniccia, ”A high speed silicon optical modulator based on a metal-oxide-semiconductor capacitor," Nature, 427, pp. 615-618 (2004).

72. A. Scherer, O. Painter, J. Vuckovic, M. Loncar, and T. Yoshie, "Photonic crystals for confining, guiding and emitting light," IEEE T. Nanotechnol., 1, pp. 4-11 (2002).

73. Q. F. Xu, V. R. Almeida, R. R. Panepucci, and M. Lipson, "Guiding and Confining Light in Void Nanostructures," Opt. Lett., 29, pp. 1206-1211 (2004).

74. T. Baehr-Jones, M. Hochberg, C. Walker, and A. Scherer, "High-Q optical resonators in slicon-on-insulator based slot waveguides," Appl. Phys. Lett., 86, 081101 (2005). 